\title{
Implication of High Grade Intraepithelial Neoplasia in Adverse Pathology after Radical Prostatectomy
}

\section{Sfoungaristos S., Perimenis P.}

Department of Urology, University Hospital of Patras, Patras, Greece Received January 4, 2012; Accepted April 11, 2012.

Key words: Prostate cancer - High grade prostatic intraepithelial neoplasia Predictor - Positive surgical margins

Abstract: The implication of high grade prostatic intraepithelial neoplasia (HGPIN) to prostate cancer aggressiveness and prognosis is conflicted. The aim of the present study was to evaluate the role of HGPIN in prediction of adverse pathology in patients undergoing a radical prostatectomy. We retrospectively analysed patients who underwent a radical prostatectomy between January 2005 and December 2010. The relationship between HGPIN and the presence of upgrade, positive surgical margins (PSM), extracapsular disease (ECD), seminal vesicle invasion (SVI) and lymph node invasion (LNI) was analysed. HGPIN predictive ability was estimated by using receiver operating characteristic curves. HGPIN was found in 160 (53.3\%) specimens. A statistically significant correlation was found between HGPIN and preoperative prostate specific antigen $(p=0.020)$ and patients' age $(p=0.025)$. No significant differences were found, regarding the presence of adverse pathological findings, between the patients with or without HGPIN, irrespective of the preoperative risk stratification. HGPIN did not reach significance for the prediction of upgrade, PSM, ECD, SVI and LNI. The presence of concomitant HGPIN and prostate cancer found not to be related with tumor aggressiveness in patients undergoing a radical prostatectomy and should not be considered as a parameter for the operative outcome prediction.

Mailing Address: Stavros Sfoungaristos, MD., Department of Urology, University Hospital of Patras, Building A, $4^{\text {th }}$ Floor, Rion, 26500 Patras, Greece; Phone: +302 610999 367; Fax: +302 610993 981; e-mail: sfoungaristosst@gmail.com 


\section{Introduction}

High grade prostatic intraepithelial neoplasia (HGPIN) has been described as a pre-malignant condition and as a precursor of prostate cancer. Actually, the two diseases share a number of similarities, like multifocality and the location mostly at the peripheral zone of the prostate (Troncoso et al., 1989; De la Torre et al., 1993). HGPIN is not a rare finding in needle biopsies and the mean incidence in prostate cores is around 9\% (Bostwick et al., 2004). The diagnosis of prostate cancer in a repeat biopsy, after a previous diagnose of HGPIN, ranges between 22 and 100\%, however, this wide range significantly downside its prognostic value (Roscigno et al., 2004; Gokden et al., 2005; Netto and Epstein, 2006; López, 2007). Irrespective of HGPIN role as a precursor of prostate cancer, its presence simultaneously with prostate malignancy is high and this is histologically proved in radical prostatectomy specimens with the incidence reaching $90 \%$ in some reports (Kovi et al., 1988; Pierorazio et al., 2007).

Despite the increase knowledge about HGPIN from a pathological perspective and the great number of studies evaluated the association of HGPIN and prostate cancer diagnosis, the potential role of HGPIN in adverse pathological outcomes of patients undergoing a radical prostatectomy has been seldom investigated. For this reason, the aim of the present study was to evaluate the prevalence of HGPIN in radical prostatectomy specimens and to identify the prognostic role of this pre-malignant disease for the presence of adverse pathological findings, in terms of positive surgical margins (PSM), extracapsular disease (ECD), seminal vesicle invasion (SVI) and lymph node invasion (LNI).

\section{Patients and Methods}

The study cohort comprised 404 consecutive patients who underwent a radical prostatectomy (open or laparoscopic) for clinically localized prostate cancer between January 2005 and December 2010 and their medical records were retrospectively analysed. Exclusion criteria were any preoperative treatment protocols, like active surveillance, hormone therapy and radiotherapy. Patients with incomplete medical records were excluded, as well.

A transrectal ultrasound-guided prostate biopsy was conducted in all patients and a minimum of 6 cores from both lobes were obtained. Bioptic cores were evaluated by our institution pathologists for the presence of malignancy and information regarding cancer grade (Gleason score) was reported.

An open or laparoscopic retropubic radical prostatectomy was performed in all patients. The procedure included the removal of the prostate gland and the seminal vesicles. A pelvic lymph node dissection was done in 206 (68.7\%) of the studied patients. The surgical specimen was then sent for histopathological examination and a report concerning the tumor grade and stage, including the presence of PSM and the presence of HGPIN, was obtained. Upgrade was defined as the presence of a higher Gleason score in the surgical specimen than that primarily found in 
biopsy. As ECD was defined any extend of tumor outside of the prostatic capsule in the periprostatic fat while the infiltration of the capsule without penetration was considered a localized disease. The invasion or not of the seminal vesicles and the dissected lymph nodes was reported, as well.

The study design had 4 objectives. The primary objective was to evaluate the correlation between HGPIN and adverse pathology, in terms of upgrade, PSM, ECD, SVI and LNI, after radical prostatectomy in the whole population of studied patients. Furthermore, we divided patients in 3 groups regarding the preoperative risk. As low risk patients were defined those with biopsy Gleason score $\leq 6$ and a preoperative prostate specific antigen (PSA) $<10 \mathrm{ng} / \mathrm{ml}$, medium risk those with Gleason score $=7$ and PSA $=10-20 \mathrm{ng} / \mathrm{ml}$ and as high risk the patients with Gleason score $\geq 8$ and PSA $>20 \mathrm{ng} / \mathrm{ml}$. The prognostic value of HGPIN was estimated in each risk group separately.

All analyses were performed by using SPSS version 17 (SPSS Inc., Chicago, IL, USA). The descriptive statistics are presented as the mean \pm standard deviation (SD) and interquartile range (IQR) for continuous variables and as the absolute and percent frequency for categorical variables.

The normality condition of the numerical variables was studied by means of the Kolmogorov-Smirnov test. None of the continuous variables had normal distribution and, for this reason, the Mann-Whitney $U$ test was used to compare means between groups. The chi-square $\chi^{2}$ test was used for categorical variables.

HGPIN was tested for its ability to predict upgrade, PSM, ECD, SVI and LNI by using receiver operating characteristic (ROC) curves. The overall performance of the ROC analysis was quantified by computing area under the curve (AUC). An area of 1 indicated perfect performance, while 0.5 indicated a performance that was not different than chance.

All tests were 2 -tailed with $\alpha$ of $p$-value of less than 0.05 to be considered as statistically significant.

\section{Results}

In total, 300 patients fulfilled the inclusion criteria and entered the analysis. The median age was 67.5 years $(66.6 \pm 6.2,9)$ and median PSA was $8.5 \mathrm{ng} / \mathrm{ml}(11.5$ $\pm 10.7,5.6)$. Based on the preoperative clinical staging, $231(77.0 \%)$ patients were T1c, 54 (18.0\%) were T2a, 12 (4.0\%) were T2b and 3 (1\%) were T2c. All studied patients were NOMO staged. HGPIN was found in 160 (53.3\%) specimens. Based on the results of the histopathological examination, PSM were identified in 127 (42.3\%) patients, ECD in 82 (27.3\%) and SVI in 45 (15.0\%) patients. A pelvic lymph node dissection was conducted in 206 patients and from those a malignant invasion was found in 26 (12.6\%) patients. An upgrade of Gleason score was noticed in 123 (41.0\%) patients. Concerning the preoperative risk stratification, $113(37.7 \%)$ patients were considered low risk, 137 (45.7\%) were medium risk and 50 (16.7\%) of them were categorized as high risk. The characteristics of the patients regarding the 
presence or not of HGPIN in radical prostatectomy specimen are seen in Table 1. A statistically significant correlation was found between preoperative PSA $(p=0.020)$ and patients' age $(p=0.025)$ and HGPIN. Table 2 is shown the prevalence of HGPIN in the whole study population and in each risk group separately. No significant differences were found, regarding the presence of adverse pathological findings, between the patients with or without HGPIN, irrespective of the preoperative risk stratification.

In the predictive ability assessment, done by the ROC analysis, HGPIN did not reach significance for the prediction of upgrade, PSM, ECD, SVI and LNI in the total population of studied patients, as well as in all risk groups (Table 3).

Table 1 - Patients' characteristics

\begin{tabular}{|c|c|c|c|}
\hline & HGPIN & non-HGPIN & $\mathrm{P}$-value \\
\hline no. of patients, n (\%) & $160(53.3)$ & $140(46.7)$ & \\
\hline age (years) & & & $0.025^{\mathrm{a} *}$ \\
\hline mean $\pm S D, I Q R$ & $65.8 \pm 6.5,9$ & $67.5 \pm 5.9,9$ & \\
\hline PSA (ng/ml) & & & $0.020^{\mathrm{a} *}$ \\
\hline mean $\pm S D, I Q R$ & $10.7 \pm 10.5,5.4$ & $12.4 \pm 10.8,8.1$ & \\
\hline Biopsy GS, n (\%) & & & $0.159^{b}$ \\
\hline$\leq 6$ & $90(55.9)$ & $71(44.1)$ & \\
\hline 7 & $58(54.2)$ & $49(45.8)$ & \\
\hline$>7$ & $12(37.5)$ & $20(62.5)$ & \\
\hline Risk group, n (\%) & & & $0.033^{\mathrm{b} *}$ \\
\hline low & $68(60.2)$ & $45(39.8)$ & \\
\hline medium & $73(53.3)$ & $64(46.7)$ & \\
\hline high & $19(38.0)$ & $31(62.0)$ & \\
\hline Pathological GS, n (\%) & & & $0.074^{b}$ \\
\hline$\leq 6$ & $66(56.9)$ & $50(43.1)$ & \\
\hline 7 & $77(55.4)$ & $62(44.6)$ & \\
\hline$>7$ & $17(37.8)$ & $28(62.2)$ & \\
\hline Pathological stage, n (\%) & & & $0.522^{b}$ \\
\hline$\leq \mathrm{T} 2 \mathrm{c}$ & $104(54.7)$ & $86(45.3)$ & \\
\hline$\geq \mathrm{T} 3 \mathrm{a}$ (including $\mathrm{N}+$ ) & $56(50.9)$ & $54(49.1)$ & \\
\hline
\end{tabular}

${ }^{a}$ Mann-Whitney U test; ${ }^{b}$ chi-square test; *statistically significant; HGPIN - high grade prostatic intraepithelial neoplasia; PSA - prostate specific antigen; GS - Gleason score; SD - standard deviation; IQR - interquartile range 
160) Prague Medical Report / Vol. 113 (2012) No. 2, p. 156-165

Table 2 - HGPIN correlation to adverse pathology

\begin{tabular}{lcccc}
\hline & & HGPIN & no HGPIN & P-value \\
\hline Total population & & & & \\
\hline number of patients, n (\%) & & $160(53.3)$ & $140(46.7)$ & \\
\hline upgrade, n (\%) & yes & $67(54.5)$ & $56(45.5)$ & 0.742 \\
& no & $93(52.5)$ & $84(47.5)$ & \\
PSM, n (\%) & yes & $72(56.7)$ & $55(43.3)$ & 0.318 \\
& no & $88(50.9)$ & $85(49.1)$ & \\
ECD, n (\%) & yes & $42(51.2)$ & $40(48.8)$ & 0.653 \\
& no & $118(54.1)$ & $100(45.9)$ & \\
SVI, n (\%) & yes & $21(46.7)$ & $24(53.3)$ & 0.331 \\
& no & $139(54.5)$ & $116(45.5)$ & \\
LNI, n (\%) & yes & $11(42.3)$ & $15(57.7)$ & 0.431 \\
& no & $110(42.3)$ & $80(57.7)$ & \\
\hline
\end{tabular}

Low risk patients

\begin{tabular}{|c|c|c|c|c|}
\hline number of patients, n (\%) & & $68(60.2)$ & $45(39.8)$ & \\
\hline \multirow[t]{2}{*}{ upgrade, n (\%) } & yes & $43(63.2)$ & $25(36.8)$ & 0.414 \\
\hline & no & $25(55.6)$ & $20(44.4)$ & \\
\hline \multirow[t]{2}{*}{ PSM, n (\%) } & yes & $21(58.3)$ & $15(41.7)$ & 0.784 \\
\hline & no & $47(61.0)$ & $30(39.0)$ & \\
\hline \multirow[t]{2}{*}{ ECD, n (\%) } & yes & $12(54.5)$ & $10(45.5)$ & 0.548 \\
\hline & no & $56(61.5)$ & $35(38.5)$ & \\
\hline \multirow[t]{2}{*}{ SVI, n (\%) } & yes & $2(50.0)$ & $2(50.0)$ & 0.672 \\
\hline & no & $66(60.6)$ & $43(39.4)$ & \\
\hline \multirow[t]{2}{*}{ LNI, n (\%) } & yes & $0(0.0)$ & $2(100.0)$ & 0.206 \\
\hline & no & $32(62.7)$ & $19(37.3)$ & \\
\hline \multicolumn{5}{|l|}{ Medium risk patients } \\
\hline number of patients, n (\%) & & $73(53.3)$ & $64(46.7)$ & \\
\hline \multirow[t]{2}{*}{ upgrade, n (\%) } & yes & $19(47.5)$ & $21(52.5)$ & 0.383 \\
\hline & no & $54(55.7)$ & $43(44.3)$ & \\
\hline \multirow[t]{2}{*}{ PSM, n (\%) } & yes & $40(61.5)$ & $25(38.5)$ & 0.066 \\
\hline & no & $33(45.8)$ & $39(54.2)$ & \\
\hline
\end{tabular}




\begin{tabular}{lcccc} 
ECD, n (\%) & yes & $24(55.8)$ & $19(44.2)$ & 0.688 \\
SVI, n (\%) & no & $49(52.1)$ & $45(47.9)$ & \\
LNI, n (\%) & yes & $10(41.7)$ & $14(58.3)$ & 0.209 \\
& no & $63(55.8)$ & $50(44.2)$ & \\
\hline High risk patients & yes & $9(69.2)$ & $4(30.8)$ & 0.212 \\
\hline number of patients, n (\%) & $53(54.6)$ & $44(45.4)$ & \\
\hline upgrade, n (\%) & yes & $5(33.3)$ & $10(66.7)$ & 0.656 \\
PSM, n (\%) & no & $14(40.0)$ & $21(60.0)$ & \\
& yes & $11(42.3)$ & $15(57.7)$ & 0.514 \\
ECD, n (\%) & no & $8(33.3)$ & $16(66.7)$ & \\
& yes & $6(35.3)$ & $11(64.7)$ & 0.777 \\
SVI, n (\%) & no & $13(39.4)$ & $20(60.6)$ & \\
& yes & $9(52.9)$ & $8(47.1)$ & 0.118 \\
LNI, n (\%) & no & $10(30.3)$ & $23(69.7)$ & \\
& yes & $2(18.2)$ & $9(81.8)$ & 0.205 \\
\hline
\end{tabular}

HGPIN - high grade intraepithelial neoplasia; PSM - positive surgical margins; ECD - extracapsular disease; SVI - seminal vesicle invasion; $L \mathrm{NI}$ - lymph node invasion; p-value was estimated by chi-square test

\section{Discussion}

Since 1987, prostatic intraepithelial neoplasia has been considered as a precursor lesion of prostate cancer (Bostwick and Brawer, 1987). This observation is supported by several histological characteristics found in cells with HGPIN that present similarities with those observed in tumorous prostate cells. Some of them include increased cellular size and variability, higher polymorphism and more prominent and atypical nuclei. Even more, both entities are mostly located in the peripheral zone of the gland. The differentiation between HGPIN and prostate cancer is histological and based on the intact cell layer and basement membrane found in cells with intraepithelial neoplasia in contrast to cancerous cells that are characterized by a destruction or absence of the membrane and changes in the glandular basal cell architecture. The above similarities in changes at the cellular level have lead to the correlation of HGPIN and prostate cancer and documented the premalignant character of intraepithelial neoplasia. The later is furthermore 
supported by the observation that the majority of patients with HGPIN lesions will develop prostate cancer within next 10 years (Bostwick et al., 2004), even though the exact time has not been exactly specified. For this reason, the latest guidelines are not recommending a repeat biopsy, in a patient with HGPIN diagnosis, earlier than 1 year. Actually, for patients diagnosed with HGPIN on extended initial core sampling (8 cores or more), a repeat biopsy within the first year is unnecessary in the absence of other clinical indicators of cancer (Lefkowitz et al., 2001; Herawi et al., 2006). In recent years, with biopsy protocols of extended cores, an overall cancer detection rate of $22-100 \%$ has been detected in patients with a previous diagnosis of intraepithelial neoplasia (Roscigno et al., 2004; Gokden et al., 2005; Netto and Epstein, 2006; López, 2007). A plurifocal HGPIN on the first biopsy set is a strong predictor for prostate cancer diagnosis on a repeat biopsy, however, it has been difficult to definitely prove which dysplastic foci of HGPIN will transform in cancer (Roscigno et al., 2004).

A concomitant presence of HGPIN in radical prostatectomy specimens has been reported to range between 86.8 and $88.4 \%$ (Kovi et al., 1988; Pierorazio et al., 2007). In our analysis, this rate was significant lower. A total of 160 (53.3\%) patients had developed both diseases. The results, regarding the presence of HGPIN in patients according to the preoperative risk stratification, have shown that high risk patients have fewer possibilities to suffer a concomitant HGPIN. Actually, HGPIN was a histological finding in $38.0 \%$ of high risk specimens, in contrast to low and medium risk patients that the incidence was higher (60.2 and 53.3\%, respectively). This observation reached statistical significance $(p=0.033)$.

In contrast to the clear and well documented relation of HGPIN and prostate cancer, the role of intraepithelial neoplasia in oncological outcome of radical prostatectomy has not been widely investigated and confirmed. With respect to upgrade of Gleason score after histological examination of the surgical specimen, it has been reported that the presence of HGPIN is one of the factors predicting a surgical Gleason score upgrade in all patients, irrespective of the preoperative risk (Moussa et al., 2009, 2010). This is not the case of our study. Our results demonstrate that there is no significant difference in upgrade incidence between patients with or without HGPIN. Furthermore, the estimation of predictive ability of HGPIN for higher tumor grade after surgery did not reveal any significance. This observation is of great importance, especially in cases that a deferred treatment strategy (active surveillance, watchful waiting) is planned for low risk patients (biopsy Gleason score $\leq 6$, preoperative PSA $<10 \mathrm{ng} / \mathrm{ml}$ ).

The theory that worse clinical characteristics of a prostatic adenocarcinoma and worse clinical outcome are correlated with the presence of concomitant HGPIN is supported by some reports. In a recent analysis of 390 patients who underwent a radical prostatectomy, the presence of concomitant HGPIN was associated with poorer prognosis in terms of poorer tumor cell differentiation, higher Gleason score and pathological stage (Auskalnis et al., 2010). From the opposite perspective, PSM 
Table 3 - Predictive accuracy of HGPIN

\begin{tabular}{lllll}
\hline & AUC & Significance & lower & upper \\
\cline { 2 - 5 } Total population & & & & \\
\hline Upgrade & 0.510 & 0.776 & 0.443 & 0.576 \\
PSM & 0.529 & 0.389 & 0.463 & 0.595 \\
ECD & 0.485 & 0.698 & 0.412 & 0.559 \\
SVI & 0.461 & 0.402 & 0.369 & 0.552 \\
LNI & 0.434 & 0.275 & 0.316 & 0.551 \\
\hline Low risk patients & & & & \\
\hline Upgrade & 0.538 & 0.491 & 0.429 & 0.648 \\
PSM & 0.486 & 0.817 & 0.372 & 0.601 \\
ECD & 0.465 & 0.612 & 0.329 & 0.601 \\
SVI & 0.447 & 0.721 & 0.157 & 0.738 \\
LNI & 0.186 & 0.135 & 0.011 & 0.362 \\
\hline Medium risk patients & & & & \\
\hline Upgrade & 0.459 & 0.453 & 0.353 & 0.566 \\
PSM & 0.579 & 0.113 & 0.483 & 0.674 \\
ECD & 0.518 & 0.730 & 0.414 & 0.623 \\
SVI & 0.430 & 0.279 & 0.304 & 0.556 \\
LNI & 0.573 & 0.394 & 0.411 & 0.734 \\
\hline High risk patients & & & & \\
\hline Upgrade & 0.467 & 0.711 & 0.292 & 0.641 \\
PSM & 0.545 & 0.587 & 0.384 & 0.706 \\
ECD & 0.480 & 0.814 & 0.310 & 0.649 \\
SVI & 0.193 & 0.445 & 0.781 \\
LNI & 0.160 & 0.175 & 0.538 \\
\hline SUC & & & & \\
\hline & & & & \\
\hline
\end{tabular}

AUC - area under the curve; $\mathrm{Cl}$ - confidence interval; other abbreviations like Table 1; results based on ROC analyses

in the same study were less commonly found in the group of patients with HGPIN. This finding is supporting the opposite that HGPIN is associated with cancer of low risk. In another large cohort of 2,133 patients, the authors reported that the presence of HGPIN in the specimens of radical prostatectomy was correlated with higher rates of tumor multifocality, perineural invasion and biochemical recurrence (Pierorazio et al., 2007). Similar results were reported in a later study of 195 radical prostatectomy specimens (Qian et al., 1997). A significant positive correlation of total volume of HGPIN and cancer volume was observed. In addition, total HGPIN volume was associated with higher pathological stage and degree of cancer differentiation. 
As we previously reported, there are some studies in the bibliography associating prostatic intraepithelial neoplasia with prostate cancer with better characteristics. In a case series which compared the clinical and histological parameters in patients with and without HGPIN, those in the HGPIN group had lower grade and volume cancers and they had fewer amounts of prostate cancer cells in their biopsy samples (López, 2007). Furthermore, the same patients were older, in contrast to our results.

The role of HGPIN in the aggressiveness of prostate cancer is controversial. Although most of the studies retrieving with this issue are reporting a positive correlation between HGPIN and prostate cancer with unfavourable cancer characteristics, there are some analyses that reporting the contrary. The results of our study showed that patients with HGPIN were younger and they had lower levels of preoperative PSA. However, there was no significant difference in the tumor grade and pathological stage between groups. Our study failed to associate pathological adverse events with HGPIN. For instance there was no higher likehood for PSM, ECD, SVI and LNI in patients with prostate cancer and concomitant HGPIN. Similar findings were shown when patients were divided into risk categories regarding the preoperative PSA and Gleason score. This comes in contrast with studies that support a better local cancer control in patients with no HGPIN, since those with HGPIN might have a higher risk of locally advanced disease and in certain circumstances it might warrant more aggressive surgical dissection, including neurovascular bundles, during the removal of the prostate (Pierorazio et al., 2007). Based on our results, we suggest that there is no benefit by the application of HGPIN as a predictor of worse pathological and clinical outcome following radical prostatectomy. Thus, it would not be correct to modify the therapeutical protocols and the surgical modifications according to the presence or not of HGPIN. Without doubts, prostatic intraepithelial neoplasia has a significant implication in prostate cancer diagnosis and should be considered when repeat biopsies are planned; however its role in prognosis of patients undergoing a radical prostatectomy is not clear and for this reason there is no need for changes in the current clinical practice until HGPIN unspecified role in prostate cancer outcome and prognosis is totally understood. Of course, the presence of HGPIN in the final pathological examination and its role in patients' prognosis are subjects that have not been thorough studied and such information would be valuable regarding potential adjuvant treatments.

Our study has some limitations that we should comment. As we reported in the patients and methods section, the present study had a retrospective design and this setting is influenced by the number and quality of patients enrolled. As a consequence, a significant number of patients were not recorded mainly because of incomplete medical records. A relatively low percentage of HGPIN incidences were found among the radical prostatectomy specimens. This observation may be biased by pathological examination. Although these cases should be excluded by the study, we cannot do this in our study since we did not know the cases that the presence or absence of HGPIN was not clearly reported. 


\section{References}

Auskalnis, S., Milonas, D., Jievaltas, M., Vaiciūnas, K., Mickevicius, A., Gudinaviciene, I. (2010) The role of high-grade prostatic intraepithelial neoplasia for biochemical relapse of prostate carcinoma after radical prostatectomy. Medicina (Kaunas) 46, 604-610.

Bostwick, D. G., Brawer, M. K. (1987) Prostatic intra-epithelial neoplasia and early invasion in prostate cancer. Cancer 59, 788-794.

Bostwick, D. G., Liu, L., Brawer, M. K., Qian, J. (2004) High-grade prostatic intraepithelial neoplasia. Rev. Urol. 6, 171-179.

De la Torre, M., Häggman, M., Brändstedt, S., Busch, C. (1993) Prostatic intraepithelial neoplasia and invasive carcinoma in total prostatectomy specimens: distribution, volumes and DNA ploidy. Br. J. Urol. $\mathbf{7 2}$, 207-213.

Gokden, N., Roehl, K. A., Catalona, W. J., Humphrey, P. A. (2005) High-grade prostatic intraepithelial neoplasia in needle biopsy as risk factor for detection of adenocarcinoma: current level of risk in screening population. Urology 65, 538-542.

Herawi, M., Kahane, H., Cavallo, C., Epstein, J. I. (2006) Risk of prostate cancer on first re-biopsy within 1 year following a diagnosis of high grade prostatic intraepithelial neoplasia is related to the number of cores sampled. J. Urol. 175, 121-124.

Kovi, J., Mostofi, F. K., Heshmat, M. Y., Enterline, J. P. (1988) Large acinar atypical hyperplasia and carcinoma of the prostate. Cancer 61, 555-561.

Lefkowitz, G. K., Sidhu, G. S., Torre, P., Lepor, H., Taneja, S. S. (2001) Is repeat prostate biopsy for high-grade prostatic intraepithelial neoplasia necessary after routine 12-core sampling? Urology 58, 999-1003.

López, J. I. (2007) Prostate adenocarcinoma detected after high-grade prostatic intraepithelial neoplasia or atypical small acinar proliferation. BJU Int. 100, 1272-1276.

Moussa, A. S., Li, J., Soriano, M., Klein, E. A., Dong, F., Jones, J. S. (2009) Prostate biopsy clinical and pathological variables that predict significant grading changes in patients with intermediate and high grade prostate cancer. BJU Int. 103, 43-48.

Moussa, A. S., Kattan, M. W., Berglund, R., Yu, C., Fareed, K., Jones, J. S. (2010) A nomogram for predicting upgrading in patients with low- and intermediate-grade prostate cancer in the era of extended prostate sampling. BJU Int. 105, 352-358.

Netto, G. J., Epstein, J. I. (2006) Widespread high-grade prostatic intraepithelial neoplasia on prostatic needle biopsy: a significant likelihood of subsequently diagnosed adenocarcinoma. Am. J. Surg. Pathol. 30, 1184-1188.

Pierorazio, P. M., Lambert, S. M., Matsukhani, M., Sprenkle, P. C., McCann, T. R., Katz, A. E., Olsson, C. A., Benson, M. C., McKiernan, J. M. (2007) High-grade prostatic intraepithelial neoplasia is an independent predictor of outcome after radical prostatectomy. BJU Int. 100, 1066-1070.

Qian, J., Wollan, P., Bostwick, D. G. (1997) The extent and multicentricity of high-grade prostatic intraepithelial neoplasia in clinically localized prostatic adenocarcinoma. Hum. Pathol. 28, 143-148.

Roscigno, M., Scattoni, V., Freschi, M., Raber, M., Colombo, R., Bertini, R., Montorsi, F., Rigatti, P. (2004) Monofocal and plurifocal high-grade prostatic intraepithelial neoplasia on extended prostate biopsies: factors predicting cancer detection on extended repeat biopsy. Urology 63, 1105-1110.

Troncoso, P., Babaian, R. J., Ro, J. Y., Grignon, D. J., von Eschenbach, A. C., Ayala, A. G. (1989) Prostatic intraepithelial neoplasia and invasive prostatic adenocarcinoma in cystoprostatectomy specimens. Urology 34, 52-56. 